\title{
Geostrophic Turbulence and Emergence of Eddies beyond the Radius of Deformation*
}

\author{
BENOIT CUSHMAN-ROISIN AND BENYANG TANG \\ Department of Oceanography, Florida State University, Tallahassee, Florida
}

(Manuscript received 15 May 1989, in final form 11 August 1989)

\begin{abstract}
Geostrophic turbulence has traditionally been studied within the framework of the classical, quasi-geostrophic equation. This equation, valid only when vertical displacements are weak, possesses a symmetry between cyclonic and anticyclonic vortices that is not present in the primitive equations. Moreover, previous studies were restricted by length scales not in excess of the deformation radius. In an attempt to advance the study of unforced geostrophic turbulence, we address here the following questions: How is the energy cascade toward longer length scales affected beyond the deformation radius? And, what is the result of the cyclonic-anticyclonic asymmetry brought on by finite vertical displacements?

Some answers are provided by numerical experiments using a generalized geostrophic equation. The energy cascade is found to come to a halt beyond the deformation radius. There, a statistical equilibrium is reached at a length scale prescribed as a combination of the deformation radius, the beta effect and the energy level of the system. Also, over the long run, one witnesses the emergence of few, large eddies, which all are anticyclonic and drift in a weaker, shorter-scale, quasi-geostrophic background. A simple theory capturing the essence of this bimodal distribution correctly predicts the bulk characteristics of the statistical equilibrium. Finally, some arguments are outlined to explain the selection of anticyclonic eddies and its relation to the statistical equilibrium.
\end{abstract}

\section{Introduction}

For numerous reasons, studies of geostrophic turbulence have relied almost exclusively upon numerical experimentation. Indeed, field observations can only be too sparse and include a number of other physical processes, laboratory experiments do not allow sufficient scale disparity between a large containing scale and a small frictional scale, and theoretical arguments can only treat bulk properties (and, even so, usually after preliminary numerical guidance). Furthermore, numerical investigations have traditionally been limited to the use of the quasi-geostrophic equation with the frequent additional assumptions of a homogeneous fluid, of a rigid surface (infinite radius of deformation), and of no beta effect (infinite earth's radius) (Rhines 1979; Mirabel and Monin 1980; McWilliams 1984; Larichev 1985, 1989; and references therein). Two facts may account for this choice. First, the two-dimensional Euler equations yield an identical equation, and, second, the quasi-geostrophic equation is solved numerically much more efficiently than the set of primitive equations from which it is derived.

* Contribution Number 281 of the Geophysical Fluid Dynamics Institute at the Florida State University.

Corresponding author address: Dr. Benoit Cushman-Roisin, Department of Oceanography, Florida State University, Tallahassee, FL 32306-3048.
Some basic results can be summarized as follows. With infinite radius of deformation and earth's radius (the Euler case), the broadening with time of the range of excited wavelengths (as expected from the adjustment to an initially narrow energy bandwidth) yields a cascade of energy toward larger scales and a cascade of enstrophy (half of the squared vorticity) toward shorter scales (Rhines 1975, 1979). The additional beta effect yields an energy-dependent length scale (here called the Rhines scale) at which zonal anisotropy prevails causing the energy cascade to slow down (Rhines 1975; Larichev 1985). What happens beyond this scale has not been addressed so far. It has been argued that a finite radius of deformation has a similar impact in slowing down the cascade of energy toward larger scales (McWilliams 1983; Larichev 1985), and again, nobody has yet addressed the question of what happens beyond the radius of deformation. Finally, Fornberg (1977), Basdevant et al. (1981) and McWilliams (1984) reported that the long-term evolution of geostrophic turbulence (even with infinite radius of deformation and earth's radius) causes saturation as successive eddy mergers yield a few, large eddies, which become so distant from one another that new collisions and mergers become increasingly rare.

The present contribution will attempt a few generalizations to the above studies of unforced geostrophic turbulence by freeing the problem from several restrictions. First, since oceanic mesoscale eddies span scales on the order of and larger than the deformation radius and since, moreover, the deformation radius $(20-50$ 
$\mathrm{km}$ ) is typically smaller than the Rhines' energy-dependent scale (200 km according to Rhines 1979), a finite deformation radius and a finite $R$ hines scale will simultaneously be retained in the formalism. These scales will act as constraints on the evolution of geostrophic turbulence.

Second, since the quasi-geostrophic equation is strictly applicable only to weak vertical displacements, a generalization of the equation will be sought to keep, at first order, some "frontal" effects, i.e., those brought by finite vertical displacements. In particular, the symmetry between cyclones and anticyclones artificially introduced by the quasi-geostrophic approximation will be broken, and the new formalism will be one step closer to the primitive equations, for which this symmetry does not exist.

The questions to be addressed here follow logically. What is the combined impact of deformable surface (finite deformation radius) and beta effect (finite Rhines' scale)? Where does the cascade of energy toward larger length scales stop, if it does stop? Finally, what is the effect of asymmetry between cyclonic and anticyclonic eddies, especially with regard to the emergence of eddies at the long term? Some answers to these questions will be provided by theoretical arguments and numerical experiments using a generalized geostrophic equation.

This generalized geostrophic equation is stated and discussed in section 2 . Then, after a few definitions (section 3), results of geostrophic turbulence below, at, and beyond the deformation radius are presented in sections 4,5 and 6 , respectively. Section 4 is but a review of classical results while sections 5 and 6 provide new elements such as the existence of a statistical equilibrium and the selection of anticyclonic vortices. A simple theory predicting the characteristics of the statistical equilibrium is proposed in section 7 , while section 8 addresses the question of the selection of anticyclonic vortices. Section 9 provides a summary of the results.

\section{A generalized geostrophic equation}

In the study of geostrophic turbulence, the ultimate choice would be to retain the complete primitive equations, but this is not possible at the present time, for several reasons. First, the primitive equations contain inertia-gravity waves, which severely restrict the time step of numerical integration for either stability or accuracy depending on the choice of an explicit or semiimplicit scheme. In addition, not one but three or more equations must be integrated. Hence, the requirements of geostrophic turbulence to resolve a wide range of length scales over a long time render the use of primitive equations prohibitively expensive. Second, the use of primitive equations raises the fundamental question of initialization of three independent fields, and it is not clear how to minimize the initial inertia-gravity waves and whether this is a reasonable choice at all. Finally, so much more is still to be learned about geostrophic turbulence by gradually enlarging the quasigeostrophic paradigm that the present authors find it wise not to use the primitive equations at this stage of knowledge, and have chosen rather to expand on the classical quasi-geostrophic formalism. (For examples of numerical modeling of eddies at geostrophic scales, the reader is referred to the recent work of Williams and Wilson 1988.)

Several generalized forms of the quasi-geostrophic equation have been proposed during the last few years (for a brief review, see Hukuda and Yamagata 1988; or Cushman-Roisin and Tang 1989). All share the advantage of containing physical processes in addition to those of the quasi-geostrophic equation while still excluding the inertia-gravity waves, and most lend themselves to numerical techniques of integration developed for the quasi-geostrophic equation.

Here, we restrict ourselves to the reduced-gravity (one moving layer) model, a rather subjective choice justified by our present purpose, namely the investigation of a great variety of horizontal length scales rather than of the effects of vertical stratification. The choice is also somewhat validated by the argument set forth by Rhines (1979) and corroborated by Gent and McWilliams (1983b, their Appendix) stating that, at length scales greater than the deformation radius, vertical-mode interactions lead to uniformization in the vertical, i.e., to barotropization of geostrophic turbulence.

With the Coriolis parameter expressed as $f_{0}+\beta_{0} y$, the reduced gravity as $g^{\prime}$, and the layer thickness as $H$ $+\eta$, where $H$ is a constant mean depth and $\eta(x, y, t)$ is the variable displacement of the interface measured positively downwards, the primitive equations yield three independent dimensional quantities: $f_{0}, \beta_{0}$ and $g^{\prime} H$. From these, two length scales can be derived, namely the planetary scale and the radius of deformation

$$
L_{\beta}=\frac{f_{0}}{\beta_{0}}, \quad L_{R}=\frac{\left(g^{\prime} H\right)^{1 / 2}}{f_{0}},
$$

respectively, and a simple dimensionless parameter emerges

$$
\alpha=\frac{L_{R}}{L_{\beta}}=\frac{\beta_{0}\left(g^{\prime} H\right)^{1 / 2}}{f_{0}^{2}} .
$$

At midlatitudes and for the open-ocean mesoscale range, typical scales are: $f_{0}=7 \times 10^{-5} \mathrm{~s}^{-1}, \beta_{0}=2$ $\times 10^{-11} \mathrm{~m}^{-1} \mathrm{~s}^{-1}, g^{\prime}=2 \times 10^{-2} \mathrm{~m} \mathrm{~s}^{-2}$, and $H=2$ $\times 10^{2} \mathrm{~m}$, providing $L_{\beta}=3500 \mathrm{~km}$ and $L_{R}=30 \mathrm{~km}$. Therefore, $L_{R}$ is much less than $L_{\beta}$, and $\alpha$ is much smaller than unity. (For the atmosphere, $L_{R} \sim 1000$ $\mathrm{km}$ and $\alpha$ is no longer small, while for the Jovian atmosphere, $\alpha$ is again small.) 
With the following scales, $L_{R}$ for $x$ and $y, 1 / f_{0}$ for time, $H$ for $\eta$ and $f_{0} L_{R}$ for the velocity components, a generalized geostrophic equation is (Cushman-Roisin and Tang 1989)

$$
\begin{aligned}
\frac{\partial}{\partial t}\left(\nabla^{2} \eta-\eta\right)+J[\eta,(1+\eta) & \left.\nabla^{2} \eta+\frac{1}{2} \nabla \eta \cdot \nabla \eta\right] \\
+\alpha(1+\eta) \eta_{x} & =0 .
\end{aligned}
$$

This equation represents a potential-vorticity balance, namely that local changes in relative vorticity $\left(\partial \nabla^{2} \eta /\right.$ $\partial t)$ and vertical stretching $(-\partial \eta / \partial t)$ are caused by advection of relative vorticity (Jacobian term) and of planetary vorticity $\left[\alpha(1+\eta) \eta_{x}\right]$. The cubic terms in the Jacobian expression and the quadratic portion of the last term are explained by the necessity to retain the full depth $(1+\eta$, in dimensionless form ) of fluid in motion, rather than its mean value, when vertical displacements are not restricted to be small. These terms, which may be called "frontal" terms, are the ones breaking the symmetry between cyclonic and anticyclonic eddies.

In the formulation of (3), the scales were chosen in relation to the few external parameters of the system in order to simplify the equation's coefficients to a maximum, but individual terms are not expected to be on the order of unity. Initial conditions will provide two additional quantities, $L$ a typical length scale (independent of $L_{R}$ and $L_{\beta}$ ) and $\delta H$ a typical vertical displacement (independent of $H$ ). Basic restrictions are $L \ll L_{\beta}$, to validate the beta-plane approximation, and $\delta H \leqslant H$, otherwise $H$ can be redefined as equal to $\delta H$.

Scrutinization of Eq. (3) then reveals that it contains two broad classes of regimes. The first one is the classical, quasi-geostrophic (QG) regime obtained when the vertical displacements are weak $(\delta H \ll H$, hence $\eta$ $\ll 1$ ) but no restriction is placed on the length scale. The prototype equation, i.e., that displaying the richest balance, is the well-known quasi-geostrophic equation

$$
\frac{\partial}{\partial t}\left(\nabla^{2} \eta-\eta\right)+J\left(\eta, \nabla^{2} \eta\right)+\alpha \eta_{x}=0,
$$

which is obtained for the particular settings $\delta H \sim \alpha H$ and $L \sim L_{R}$. The other class contains the frontal geostrophic (FG) dynamics, obtained when the length scale is larger than the deformation radius $\left(L_{R} \ll L\right.$ $\ll L_{\beta}$ ) but no restriction is placed on the vertical displacements. The prototype equation is

$$
\begin{aligned}
-\frac{\partial \eta}{\partial t}+J\left[\eta,(1+\eta) \nabla^{2} \eta+\right. & \left.\frac{1}{2} \nabla \eta \cdot \nabla \eta\right] \\
& +\alpha(1+\eta) \eta_{x}=0,
\end{aligned}
$$

which is obtained for the particular settings $\delta H \sim H$ and $L \sim L_{R}{ }^{2 / 3} L_{\beta}{ }^{1 / 3}=\alpha^{-1 / 3} L_{R}$ (Cushman-Roisin 1986).
Each class contains a family of subregimes, for which the equations are simplications of Eqs. (4) or (5). If both assumptions of weak vertical displacements $(\delta H$ $\ll H)$ and long length scales $\left(L_{R} \ll L\right)$ are made simultaneously then the resulting equation is

$$
-\frac{\partial \eta}{\partial t}+J\left(\eta, \nabla^{2} \eta\right)+\alpha \eta_{x}=0
$$

which can be considered as the common denominator to both classes of regimes. If neither assumption is made (then $\delta H \sim H$ and $L \sim L_{R}$ ), the Rossby number is not small, the system is not nearly geostrophic, Eq. (3) cannot be stated, and one must return to the full set of the primitive governing equations.

In Eqs. (4), (5) as well as (6), the diagnostic terms consist of a Jacobian and a term with coefficient $\alpha$, the former representing advection and the latter the beta effect. Both will be on the same order of magnitude only if a constraint between $L$ and $\delta H$ exists, viz. $\delta H /$ $H=\alpha\left(L / L_{R}\right)^{3}$. The particular value of the length scale for which this relationship holds is heretofore called the Rhines scale:

$$
L_{E}=L_{R}\left(\frac{\delta H}{\alpha H}\right)^{1 / 3}=\left(L_{R}^{2} L_{\beta} \frac{\delta H}{H}\right)^{1 / 3} .
$$

The connection between this definition and the scale noted by Rhines (1975) will be made shortly, but it can already be remarked that this length depends on the amplitude, $\delta H$, of the motion and hence on the energy in the system. (The subscript ${ }_{E}$ has been chosen to emphasize this energy dependency.)

Figure 1 recapitulates in the $(L, \delta H)$ parameter space the numerous constraints on the scales and the various geostrophic regimes (see Cushman-Roisin and Tang 1989 , for additional details). Midlatitude mesoscale eddies and rings of the ocean, whose scales are indicated by the shaded area of Fig. 1, do not all fall in the geostrophic formalism but those that do mostly fall in the parameter range $L_{R} \leqslant L \leqslant \alpha^{-1 / 3} L_{R}$ and $\delta H \leqslant H$. This latter range will be the object of the following numerical experiments.

To every regime corresponds a time scale (CushmanRoisin and Tang 1989). The general tendency is for that time scale to vary from the inertial period (at the prohibited limit $L=L_{R}, \delta H=H$ ) toward larger values with increasing length scale and/or decreasing amplitude. Interestingly enough, the time scale saturates at a value of $1 / \alpha^{2} f_{0}$ at the intermediate geostrophic regime obtained for $L=\alpha^{-1 / 3} L_{R}, \delta H=\alpha^{2 / 3} H$ (Williams and Yamagata 1984). With the numbers quoted above for midlatitude conditions, this corresponds to periods of several years, and, for the ocean, additional processes such as heat flux, evaporation and precipitation can no longer be ignored. In tropical latitudes where the thermocline is sharper and the planetary scale shorter, the intermediate-geostrophic time scale may be as short as several months (Matsuura and Yamagata 1982), 

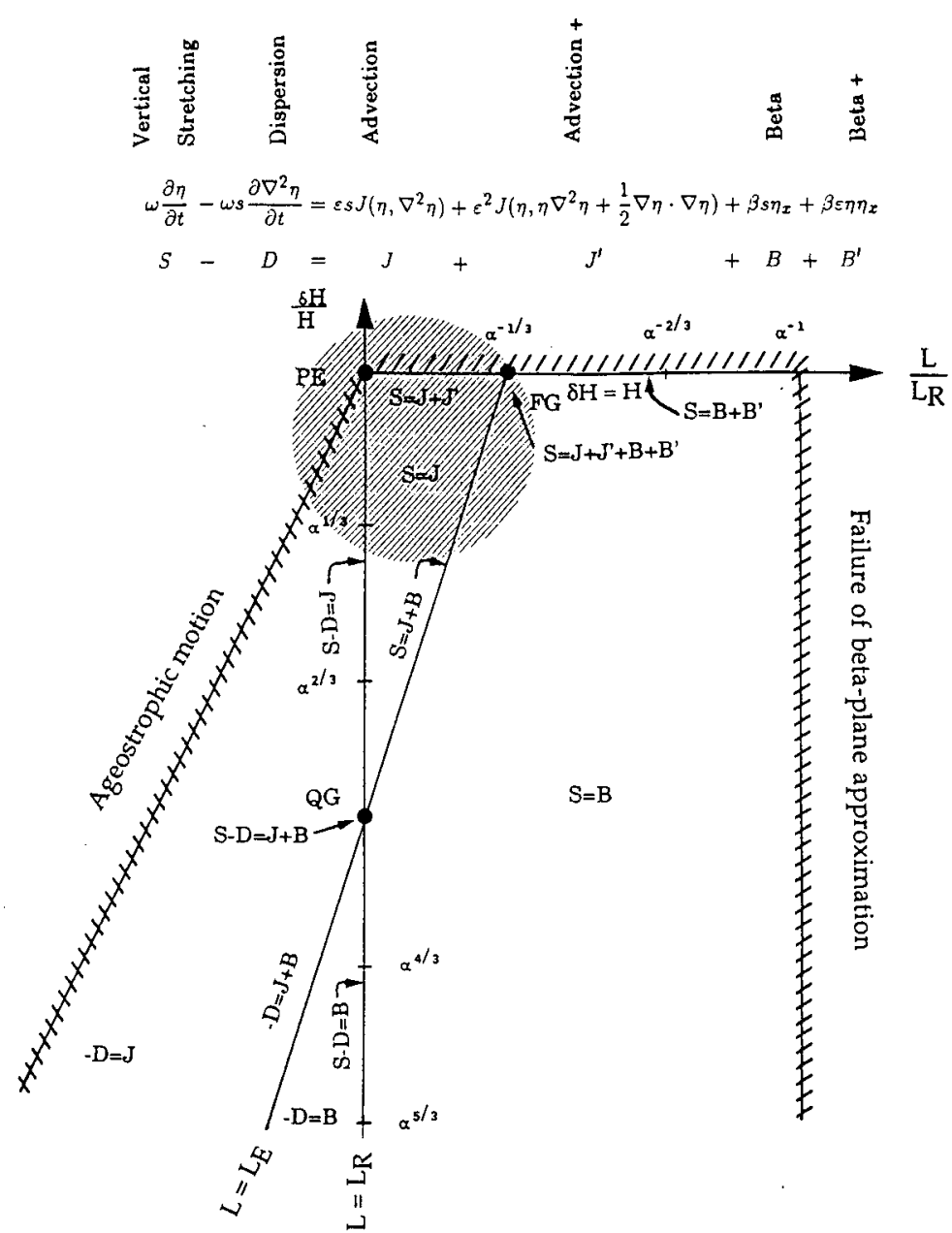

FIG. 1. The various geostrophic regimes obtained from Eq. (3). The abbreviations PE, QG and FG stand, respectively, for the primitive equations (excluded from the geostrophic regimes), the classical quasi-geostrophic regime, and the frontal geostrophic regime. The parameters are $L / L_{R}$, the ratio of the eddy length scale to the deformation radius, and $\delta H / H$, the ratio of the eddy amplitude to the mean layer depth. The hatched areas fall outside the scope of the generalized geostrophic equation, while the shaded region indicates the scales of oceanic mesoscale eddies and rings.

but then wind forcing may be crucial (Yamagata and Umatani 1990). Being concerned here solely by unforced, midlatitude turbulence, we will not further consider the intermediate geostrophic regime (lowerright portion of Fig. 1).

In the numerical experiments reported below, the full equation ( 3 ) is being solved in a doubly periodic, square domain and for various initial conditions. For mathematical convenience, we adopt here the value $\alpha$ $=1 / 64$, which is an integer power of $1 / 2$.

Equation (3) has two invariants. An integration over the domain yields the law of volume (mass) conservation

$$
\frac{d V}{d t}=0, \quad V=\iint \eta d x d y
$$

while multiplying by $\eta$ and then integrating yields the law of energy conservation

$$
\frac{d E}{d t}=0, \quad E=\frac{1}{2 A} \iint\left(\eta^{2}+|\nabla \eta|^{2}\right) d x d y=0 .
$$

In the latter integral, the first and second terms can be interpreted as the potential and kinetic energy, respectively. (The domain area, $A$, is introduced for convenience.)

The enstrophy is defined as half of the total squared (linearized) potential vorticity (divided here by the domain area, $A$ )

$$
Z=\frac{1}{2 A} \iint\left(\nabla^{2} \eta-\eta\right)^{2} d x d y
$$


and its evolution equation is

$$
\frac{d Z}{d t}=\frac{1}{2 A} \iint|\nabla \eta|^{2}\left[J\left(\eta, \nabla^{2} \eta\right)+\alpha \eta_{x}\right] d x d y .
$$

The right-hand side of (10b) is not zero in general, and enstrophy is thus not conserved by equation (3). However, careful inspection reveals that, for the class of quasi-geostrophic regimes [see Eq. (4)], enstrophy as defined by (10a) is conserved while, for the class of frontal geostrophic regimes [see Eq. (5)], a simplified version of enstrophy [expression (10a) without the $\nabla^{2} \eta$ term, which is then negligible] is also conserved. In other words, each regime has its own conserved enstrophy, but the generalized equation does not. If the system falls in the QG regime, the right-hand side of (10b) is nonzero only because of the small and negligible terms specific to the FG regime, and vice versa. Hence, enstrophy as defined by (10a) is not exactly but almost conserved by equation ( 3 ).

The generalized geostrophic equation, Eq. (3), is not new. In fact, it first appeared in studies of plasma physics where the magnetic field plays a role similar to the Coriolis force (Petviashvili 1980), and has since appeared in one form or another in the geophysicalfluid literature. A discussion of the analogy between plasma drift waves and oceanic Rossby waves can be found in Meiss and Horton (1983) and Weinstein (1983).

After some elementary scale considerations and an ad hoc development of the intermediate-geostrophic equation by Charney and Flierl (1981), Williams and Yamagata (1984) and then Williams (1985) set out to derive a generalized geostrophic equation. A first equation is our Eq. (3) with the addition of several terms, some always negligible and some permitting extension to low latitudes where $\beta$-plane geostrophy supersedes $f$-plane geostrophy. In the same article, Williams (1985) then derived a reduced, midlatitude version of his equation, which includes our Eq. (3). That equation cannot be integrated numerically because of a nonlinearity among the time-derivative terms. As with our equation, volume and energy are conserved, while enstrophy is not.

Pursuing this line of work, Hukuda and Yamagata (1988) very recently explored several variations of the equation to determine whether the addition of small, negligible but carefully chosen terms might lead to an enstrophy conservation law. Although no general proof was proposed, their results indicate a trade-off: either energy or enstrophy can be conserved but not both simultaneously. This conclusion is somewhat puzzling, for the set of primitive equations from which the generalized equation is derived conserves both energy and enstrophy. The reason probably lies in the fact that the generalized geostrophic equation is a hybrid formulation containing two distinct families of regimes, each having its separate and not-overlapping expressions for energy and enstrophy. In other words, the generalized geostrophic equation does not converge uniformly as the Rossby number tends toward unity.

With a different approach, Gent and McWilliams (1983a, 1983b) derived so-called balance equations. These are obtained by introducing two new variables, a streamfunction and a velocity potential and by assuming that the former is much larger than the latter (i.e., at first approximation the horizontal flow is nondivergent ). In the general context of continuous stratification, a baroclinic twisting term masks the frontal terms retained in the present, reduced-gravity model, and the dynamics are quite different. Close examination reveals that the reduction of the Gent-McWilliams balanced equations to the reduced-gravity system does not yield all terms of our formalism; namely, it ignores the quadratic correction to the beta term. Again, as for our equation, enstrophy is not strictly conserved.

To guarantee that volume and energy together with enstrophy be conserved, Salmon (1985) proceeded to derive a family of geostrophic formalisms from variations on a Hamiltonian principle. In the category of interest to us (i.e., wide range of scales), the formalisms are sets of equations whereby the main, prognostic equation is coupled to one or more diagnostic equations, the latter being elliptical with nonconstant coefficients. A very similar set of equations (perhaps identical to one of Salmon's formalisms) was derived by Sutyrin and Yushina (1986), who used a straightforward perturbation technique. Such systems of coupled equations offer the advantage of strict conservation of volume, energy and enstrophy (and may well be the only formalisms to do so). However, they suffer from not being amenable to standard spectral techniques of integration, which are desirable for their accuracy/ economy properties so crucial when a large spectrum of scales is contemplated.

For the numerical integration, we use a spectral method for its reputedly high ratio of accuracy versus number of calculations, preventing ourselves from using Salmon's equations. In the following results obtained by using Eq. (3), we noted that the enstrophy defined by (10a) varied by a negligible amount (less than $1 \%$, if at all perceptible) over long integration times (on the order of 100 times the dynamical time scale). Hence, despite its lack of an enstrophy-conservation law, the equation that we propose here to integrate is nonetheless a prototype for the study of geostrophic turbulence beyond the classical quasi-geostrophic formulation.

\section{A few definitions}

At any time during the evolution of the height field, a characteristic amplitude, $\delta H$, and a characteristic length scale, $L$, can be defined with 
$\delta H^{2}=\frac{1}{A} \iint \eta^{2} d x d y,\left[\frac{\delta H}{L}\right]^{2}=\frac{1}{A} \iint|\nabla \eta|^{2} d x d y$

where the integration extends over the rectangular domain, the area of which is $A$. The latter definition differs from the energy-weighted length scale used by Rhines $(1975,1979)$ and many others. The reason is that, in Rhines' classical approach, the quasi-geostrophic equation is so simplified that the energy is mostly kinetic, while here the importance of potential energy would render a generalization of that definition awkward and ill suited.

As geostrophic turbulence evolves, interactions modify the turbulent field and its overall characteristics. Consequently, the quantities $\delta H$ and $L$ are time-dependent, and a basic question concerns their evolution and eventual limit: Do preferential values exist toward which $\delta H$ and $L$ evolve as the system adjusts from the initial conditions to some presumed statistical equilibrium?

One constraint can be immediately stated. With definitions (9) and (11), the total energy (divided by the domain area) is
$E=\frac{1}{2 A} \iint\left(\eta^{2}+|\nabla \eta|^{2}\right) d x d y=\frac{1}{2} \delta H^{2}\left[1+\frac{1}{L^{2}}\right]$,

and since $E$ is conserved, $\delta H$ and $L$ are not independent. As a result, the point $(L, \delta H)$ representing the bulk characteristics of the system must evolve along energy lines, as defined by (12) and displayed on Fig. 2.

For length scales much shorter than the deformation radius, energy is dominated by the kinetic energy, and (12) reduces to $E=\delta H^{2} / 2 L^{2}$, or, with scales, $E$ $=g^{\prime 2} \delta H^{2} / 2 f_{0}^{2} L^{2}$. If, following Rhines (1975), one defines an average velocity $U$ such as $E=U^{2} / 2, \delta H$ is found to be related to $L$ via $g^{\prime} \delta H=f_{0} L U$, and definition (7) becomes

$$
L_{E}=\left[\frac{U}{\beta_{0}}\right]^{1 / 2},
$$

an expression in which the reader may recognize the scale introduced by Rhines. When the length scale is not short compared to the deformation radius, kinetic energy no longer dominates the energy budget, the quantity $U$ is not an invariant, and (13) becomes ill

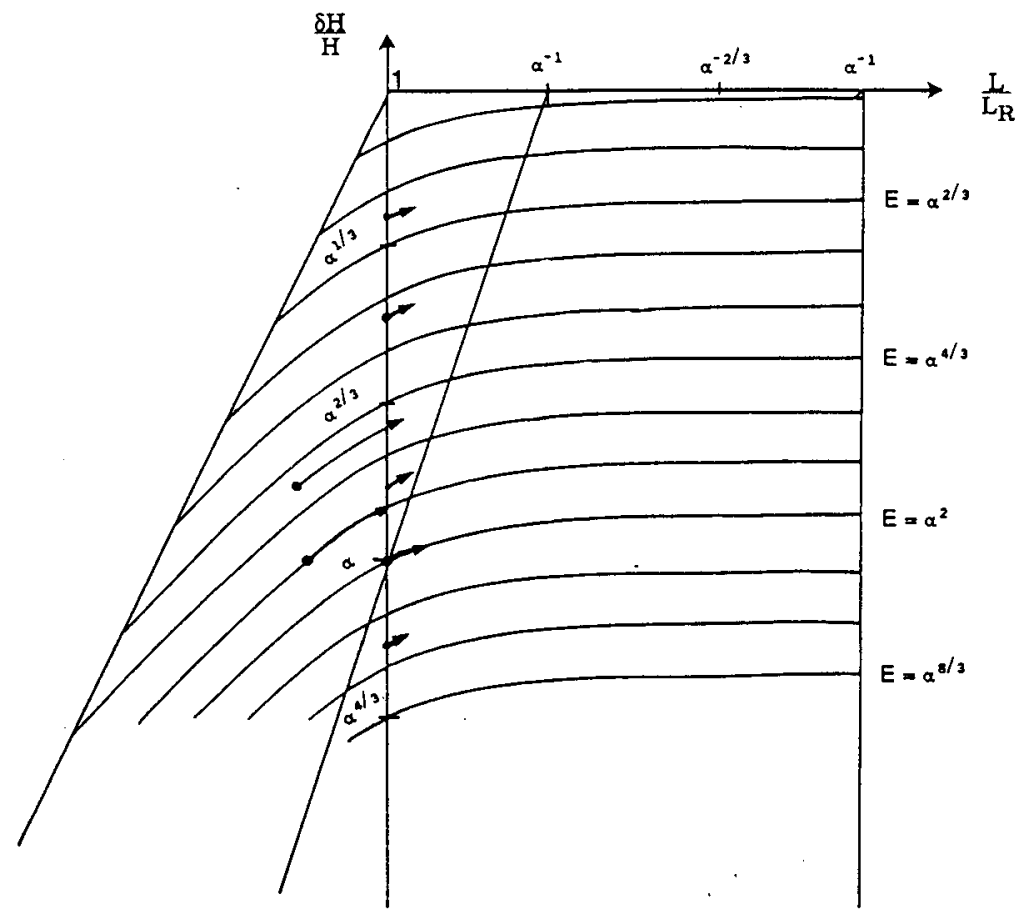

Fig. 2. Lines of constant energy in the parameter space. As geostrophic turbulence evolves, the point $(L, \delta H)$ moves along the line corresponding to the initial energy level. The arrows show the evolution for a series of numerical experiments at length scales up to the deformation radius. Note the drift toward longer length scales, even at the deformation-radius scale. 
suited. On the contrary, definition (7) resulting from a comparison between advection and beta terms remains meaningful and practical at all scales.

A useful quantity is the following

$$
W=\frac{\left[\iint \eta^{2} d x d y\right]\left[\iint\left(\nabla^{2} \eta\right)^{2} d x d y\right]}{\left[\iint|\nabla \eta|^{2} d x d y\right]^{2}},
$$

which measures the width (spread) of the spectrum of $\eta$. Indeed, the Schwartz inequality implies $W \geqslant 1$, and $W=1$ if and only if $\eta$ consists of a single wavelength $\left(\nabla^{2} \eta=-k^{2} \eta\right)$. The greater the number of excited wavelengths, the larger $W$.

\section{Below the deformation radius}

The classical studies of geostrophic turbulence by Rhines $(1975,1979)$ begin with the simplified equation

$$
\frac{\partial}{\partial t} \nabla^{2} \eta+J\left(\eta, \nabla^{2} \eta\right)=0
$$

obtained from (3) with the assumption of quasi-geostrophy $(\delta H \ll H)$, infinite deformation radius ( $L$ $\ll L_{R}$ ) and infinite earth radius ( no beta effect, $L \ll L_{E}$ ). On Fig. 1, this regime corresponds to the lower-left sector. The equation conserves kinetic energy (potential energy is neglected ) and a simplified form of enstrophy:

$E=\frac{1}{2 A} \iint|\nabla \eta|^{2} d x d y, Z=\frac{1}{2 A} \iint\left(\nabla^{2} \eta\right)^{2} d x d y$

respectively.

With (11) and (14), the spectrum width can be expressed in terms of these invariants and $\delta H$ or $L$ :

$$
W=\frac{Z \delta H^{2}}{2 E^{2}}=\frac{Z L^{2}}{E} .
$$

Then, if one assumes that mutual interactions between waves and eddies can only spread the spectrum $(d W /$ $d t>0$ ), it follows that both the amplitude and the length scale must increase ( $d \delta H / d t>0, d L / d t>0)$. This is the well-known property of energy cascade toward longer scales. On Fig. 2, the $(L, \delta H)$ point rises from the lower left sector along the energy line set by the initial conditions, until it eventually hits either the line ( $L=L_{E}$ ) or the line $\left(L=L_{R}\right)$, depending on the energy level. The first case occurs for sufficiently low energies $\left(E<\alpha^{2}\right)$ and implies that the new term to be added in the equation is the beta term $\left(\alpha \eta_{x}\right)$, while the second case occurs for higher energies $\left(\alpha^{2}<E<1\right)$ and brings in the vertical stretching term $(-\partial \eta / \partial t)$.
Although oceanic eddies have energies that would place them in the latter case, Rhines (1975) studied the former possibility, and investigated the modified equation

$$
\nabla^{2} \eta_{t}+J\left(\eta, \nabla^{2} \eta\right)+\alpha \eta_{x}=0
$$

which has the same invariants (16) as equation (15). It follows directly that, if the spectrum keeps widening, the length scale will continue to increase. However, Rhines convincingly argued that the organization of Rossby waves should slow down if not arrest the energy cascade.

The spontaneous emergence of a few large eddies from the initial multitude of smaller eddies (Fornberg 1977; Basdevant et al. 1981; McWilliams 1984) yields another argument. As ever fewer eddies emerge, distances between them increase, and the likelihood of future mergers decreases, perhaps halting altogether the progression toward larger scales ( see also Rhines 1975, for comments on the sparse eddy case). Hence, if the initial length scale is sufficiently small, geostrophic turbulence may be virtually arrested before the Rhines scale or the deformation radius can be reached.

A very important question, however, remains unanswered. Oceanic eddies have typical radii on the order of or larger than the deformation radius and typical vertical interfacial displacements of order unity, and they simultaneously feel the beta effect. Consequently, their dynamics do not correspond to either equation (15) or (18), and oceanic geostrophic turbulence cannot be thought of as the long-term evolution of geostrophic turbulence $a$ l $l a$ Rhines or McWilliams. What then can be said of its properties?

\section{At the radius of deformation}

When the length scale is on the order of the deformation radius, previous studies are rather tacit (see McWilliams 1983; Larichev 1985). However, it can be proved that the cascade toward longer scales does not stop there but merely slows down. A proof follows.

At the scale of the deformation radius, the governing equation is

$$
\frac{\partial}{\partial t}\left(\nabla^{2} \eta-\eta\right)+J\left(\eta, \nabla^{2} \eta\right)+\alpha \eta_{x}=0,
$$

where advection (Jacobian term) or the beta effect dominate depending upon whether the energy is high $\left(\alpha<\delta H / H<1, \alpha^{2}<E<1\right)$ or low $(\delta H / H<\alpha, E$ $\left.<\alpha^{2}\right)$. Irrespective of which of the last two terms is dominant, the energy and enstrophy invariants are (per unit area)

$$
E=\frac{1}{2 A} \iint\left(\eta^{2}+|\nabla \eta|^{2}\right) d x d y=\frac{1}{2} \delta H^{2}\left(1+\frac{1}{L^{2}}\right)
$$




$$
\begin{aligned}
Z & =\frac{1}{2 A} \iint\left(\nabla^{2} \eta-\eta\right)^{2} d x d y \\
& =\frac{1}{2 A} \iint\left(\nabla^{2} \eta\right)^{2} d x d y+\frac{\delta H^{2}}{L^{2}}+\frac{\delta H^{2}}{2} .
\end{aligned}
$$

The spectrum width, defined by (14), can be written as

$$
W=\frac{Z}{E} L^{2}\left(1+L^{2}\right)-\left(1+L^{2}\right)^{2}+1,
$$

after using (11) and (21) to replace the integrals by terms involving $\delta H, L$ and $Z$ and then eliminating $\delta H$ with (20). The assumption of a widening spectrum $(d W / d t>0)$ yields

$$
2 L\left[\frac{Z}{E}\left(1+2 L^{2}\right)-2\left(1+L^{2}\right)\right] \frac{d L}{d t}>0,
$$

while the property $W \geqslant 1$ provides

$$
\begin{gathered}
\frac{Z}{E} L^{2}\left(1+L^{2}\right) \geqslant\left(1+L^{2}\right)^{2} \\
\frac{Z}{E} \geqslant \frac{1+L^{2}}{L^{2}}=\frac{2\left(1+L^{2}\right)}{2 L^{2}}>\frac{2\left(1+L^{2}\right)}{1+2 L^{2}} .
\end{gathered}
$$

The last inequality in (24) implies that the bracketed quantity in (23) is positive and thus

$$
\frac{d L}{d t}>0
$$

i.e., the drift toward longer length scales still goes on at the scale of the deformation radius.

A series of seven numerical experiments were performed with Eq. (3) using the spectral method. The progress of $(L, \delta H)$ is traced by the arrows of Fig. 2, which all show the drift toward longer length scales. As the integration time of each run was a fixed multiple of its intrinsic time scale, a shorter arrow means a slower drift. Some additional information on one of the runs (that corresponding to the lowest arrow of Fig. 2) is provided on Fig. 3. The initial characteristics are those of the classical quasi-geostrophic regime (QG point of Fig. 1, with $L=L_{R}, \delta H=\alpha H$, and thus $E$ $\left.=\alpha^{2}\right)$. The initial spectrum is centered about $k=4$ (four waves in the $64 \times 64$ domain) and has a spread in wavenumbers of about $20 \%$. Comparison of the upper two panels and of the initial and final spectra clearly reveals the drift toward longer length scales. Despite the retention of negligible but not neglected terms that preclude the existence of a rigorous enstrophy-conservation law, enstrophy is very well conserved, and so is energy, as it must be. Although the narrowness of the domain allows only four initial waves in each direction, there is an indication that emergence of eddies is occurring.

\section{Beyond the radius of deformation}

Typical numbers for oceanic mesoscale eddies and rings suggest $L_{R}=30$ to $50 \mathrm{~km}, L=50$ to $200 \mathrm{~km}, H$ $=50$ to $500 \mathrm{~m}$, and $\delta H=100$ to $400 \mathrm{~m}$. This positions us in the parameter space in the triangle defined by the three centers PE, QG and FG of Fig. 1, where the length scale is on the order of or slightly larger than the deformation radius $\left(L \geqslant L_{R}\right)$, the amplitude is almost frontal $(\delta H \leqslant H)$, and the beta effect is noticeable ( $L$ $\left.\leqslant L_{E}\right)$.

Within the triangle and sufficiently away from its boundaries, the governing equation reduces to

$$
-\frac{\partial}{\partial t} \eta+J\left(\eta, \nabla^{2} \eta\right)=0
$$

Potential energy dominates kinetic energy and the expression for the enstrophy degenerates to the integral of $\eta^{2} / 2$, such that

$$
E=Z=\frac{1}{2 A} \iint \eta^{2} d x d y
$$

is a single invariant. In addition, the kinetic energy

$$
K=\frac{1}{2 A} \iint|\nabla \eta|^{2} d x d y,
$$

is an independent invariant. From the conservation of $E$ and $K$, viz. (26) and (27), and the definitions of $\delta H$ and $L$, viz. (11), it immediately follows that $\delta H$ and $L$ are invariants. Hence, at first order, there is no cascade of energy or enstrophy in this parameter regime, and this may account for why the cascade of energy toward longer length scales noticeably slows down as $L$ approaches and then surpasses the deformation radius.

For the ocean, $\alpha=L_{R} / L_{\beta}$ is about $30 \mathrm{~km}$ over 3500 $\mathrm{km}$ so that $\alpha^{1 / 3}(\sim 0.2)$ is not so small. Consequently, the regime triangle under consideration is not sufficiently wide for Eq. (25) to hold with reasonable accuracy, and additional, small but not negligible, terms ought to be retained. The presence of these will control the energy cascade.

Three new terms must be included, one coming from each regime bordering the triangle under consideration. The proximity to the line $L=L_{R}$ brings in the dispersion term $\nabla^{2} \eta_{t}$, which has been found to induce a slow energy cascade toward longer length scales (section 5 ). The proximity to the line $L=L_{E}$, the Rhines scale, adds the linearized beta term $\alpha \eta_{x}$; but this term does not modify invariants (26) and (27) and, alone, is therefore unable to alter the energy cascade. Finally, the proximity to the line $\delta H=H$ brings in the cubic terms $J\left(\eta, \eta \nabla^{2} \eta\right)$ and $J\left(\eta, \frac{1}{2} \nabla \eta \cdot \nabla \eta\right)$, the impact of which cannot be readily ascertained.

A number of numerical experiments were performed to investigate the behavior of geostrophic turbulence 

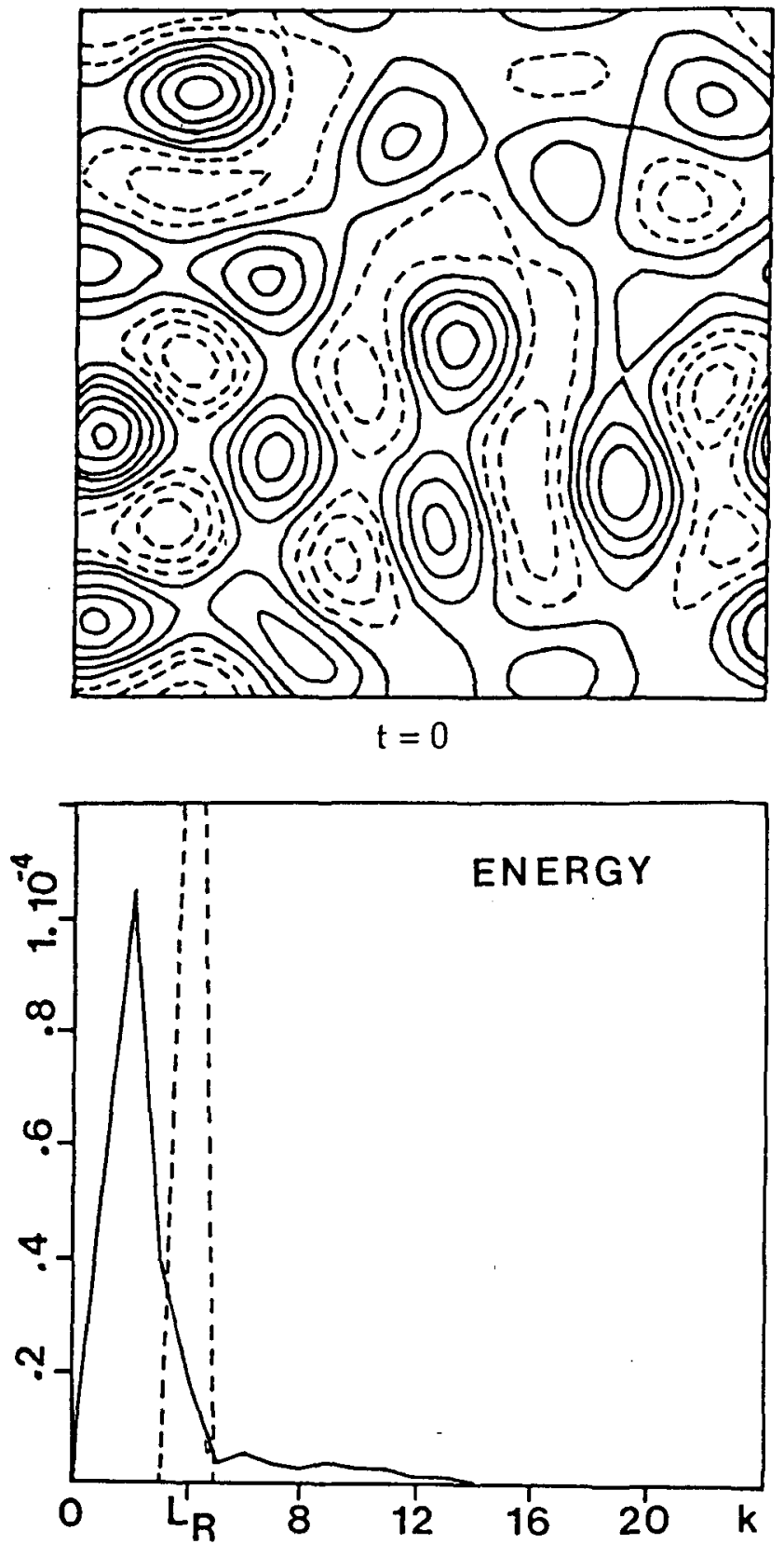

beyond the radius of deformation. The results are recapitulated on Fig. 4, where arrows trace the evolution of $L$ and $\delta H$ for the runs during which these values drifted noticeably and where crosses mark those values of $L$ and $\delta H$ for the runs during which they remain almost constant. A definite pattern emerges: for length scales less than, equal to or slightly larger than the deformation radius, $L$ drifts toward larger values, while for length scales substantially beyond the deformation radius, $L$ evolves toward smaller values. There thus exists a line separating both regions and along which

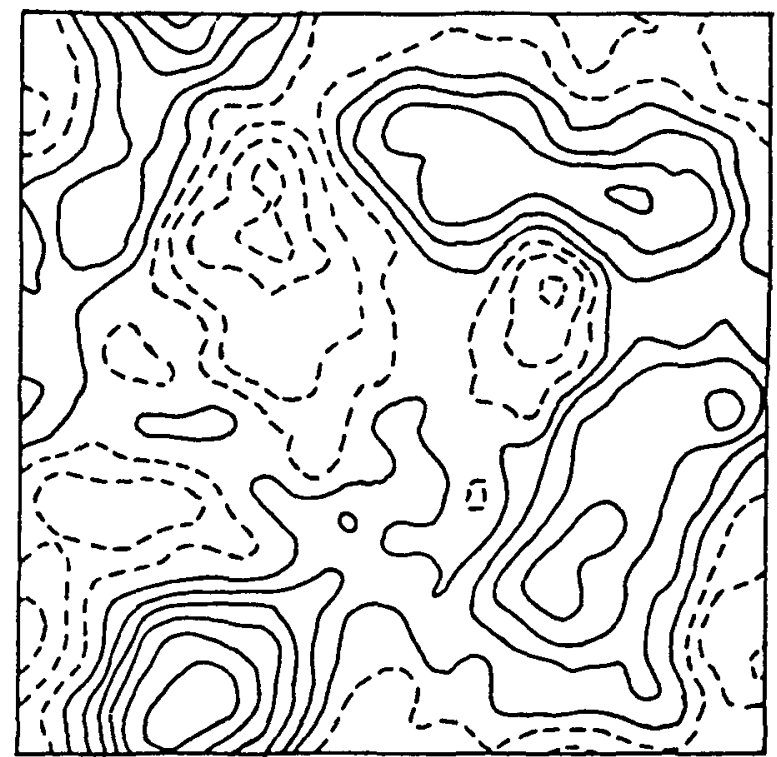

$t=150000=234$ time scales

Fig. 3. Numerical results for a typical quasi-geostrophic run. Initially, the parameters are $L=L_{R}, \delta H=\alpha H, E$ $=\alpha^{2}$. The upper panels compare the $\eta$ field at the initial and final times $(t=0$ and $t=15,000$ or 234 intrinsic time scales). The lower-left panel traces the evolution of $L$, $\delta H, E, Z$ and $W$, while the lower-right panel displays the initial and final spectra.
$L$ does not change. In other words, the classical energy cascade toward longer scales halts at scales beyond the deformation radius, and a reverse cascade toward shorter scales occurs beyond that limit.

Points along the dividing line appear as attractors, setting a preferential length scale for each energy level, and we now ask what balance among physical processes does this line represent. In other words, what are the opposing mechanisms responsible for the arrest of the energy cascade? To answer this question, a detailed examination of the numerical results is in order. 


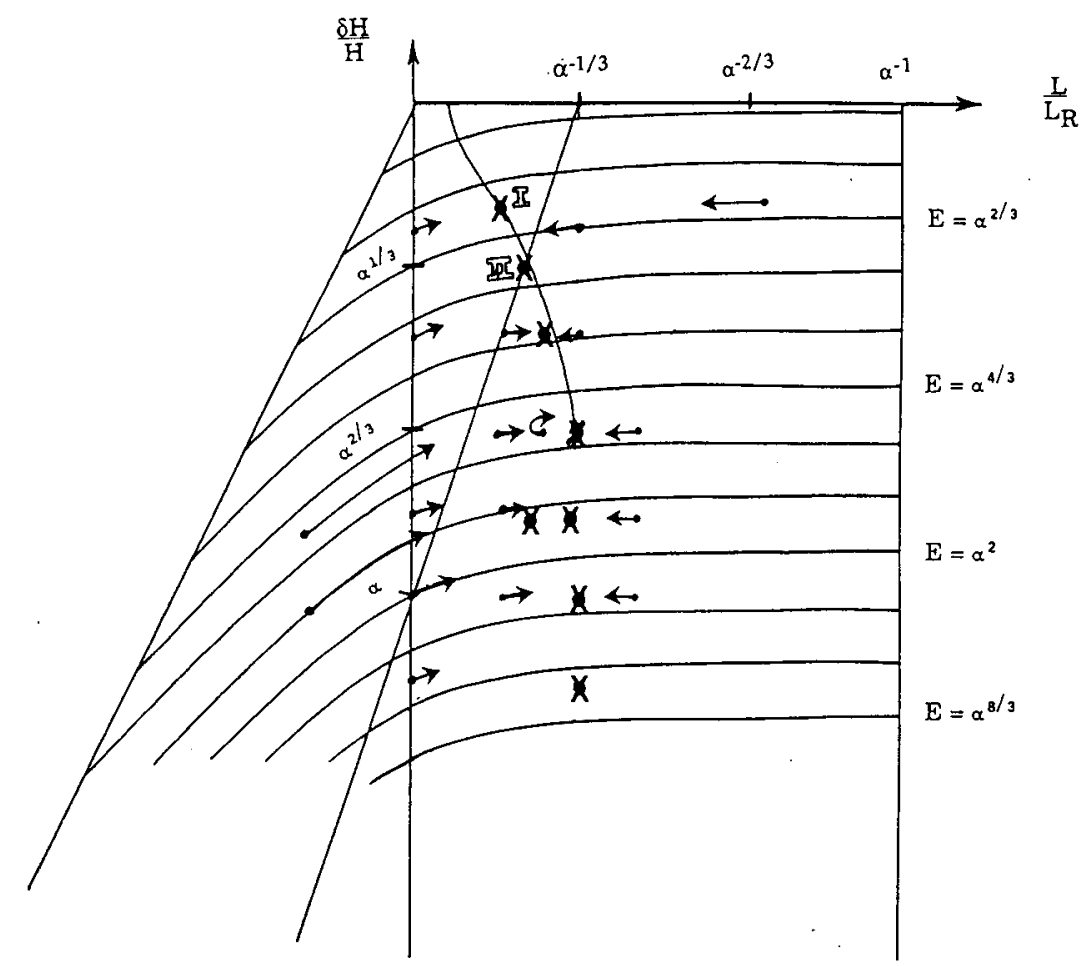

FIG. 4. Recapitulation of all numerical experiments performed for this study. Arrows trace the evolution of $L$ and $\delta H$ when these values drifted noticeably during the course of the calculations. Crosses mark values of $L$ and $\delta H$ for runs during which they remained almost constant, while the $S$-shape curve is the theoretical prediction for the set of those points. Plots corresponding to runs I and II are provided by Figs. 5 and 6 .

Figure 5 reports the results for run I as marked on Fig. 4. During the course of this run, the values of $L$ and $\delta H$ are found to be almost constant ( $L$ decreases very slightly, by less than $9 \%$ during 94 intrinsic time scales, and the variation of $\delta H$ is imperceptible), and this run can be considered as representative of one on the dividing line. Contrasting the upper two panels of Fig. 5, from initial to final times, we notice a marked decrease in the number of eddies (attributable to successive mergers as revealed by intermediate plots not reported here). The new eddies are somewhat larger than the initial eddies and, most strikingly, all are anticyclonic. The background, in which these eddies evolve, is of smaller scale than the initial turbulent field, so as to preserve the overall, mean length scale. Although remarkable, the emergence of eddies from the turbulent flow comes as no surprise since McWilliams (1984) already reported this tendency in the quasigeostrophic context. The novel aspect here is the selection of emerging eddies: not a single cyclonic eddy has emerged. This property stands in contrast to McWilliams' results where neither polarity was favored and the late stages of turbulence contain about equal proportions of cyclonic and anticyclonic eddies. Of course, in that study, the symmetry of the quasi-geo- strophic equation could not discriminate between either species.

The shattered cyclonic eddies make a smaller-scale, turbulent background in which large-scale, coherent anticyclones evolve, and the resulting distribution reveals a bimodal character. This bimodality is also corroborated by the shape of the spectrum at the ending time (lower panel of Fig. 5). There, we notice a peak around wavenumber $k=2$ (i.e., two waves per domain width, or $L=4 L_{R}=\alpha^{-1 / 3} L_{R}$ for this run ) where most of the energy is concentrated, and a tail with wavenumbers ranging from 6 to 14 , approximately centered over the deformation radius $(k=8)$. Evidently, the peak corresponds to the large, energetic eddies, and the tail to the smaller-scale, weaker background. Moreover, the amplitude of the eddies ( $\delta H$ close to $H$ ) places them in the frontal-geostrophic regime, while the amplitude ( $\delta H \ll H)$ and the length scale $\left(L \sim L_{R}\right)$ of the background classify the latter as quasi-geostrophic.

Figure 6 displays similar information for run II of Fig. 4. The initial length scale is larger, the energy is smaller, and the numerical resolution is doubled, compared to run I. Again, the average length scale, $L$, and the average amplitude, $\delta H$, remain almost constant throughout the calculations, and similar findings are 

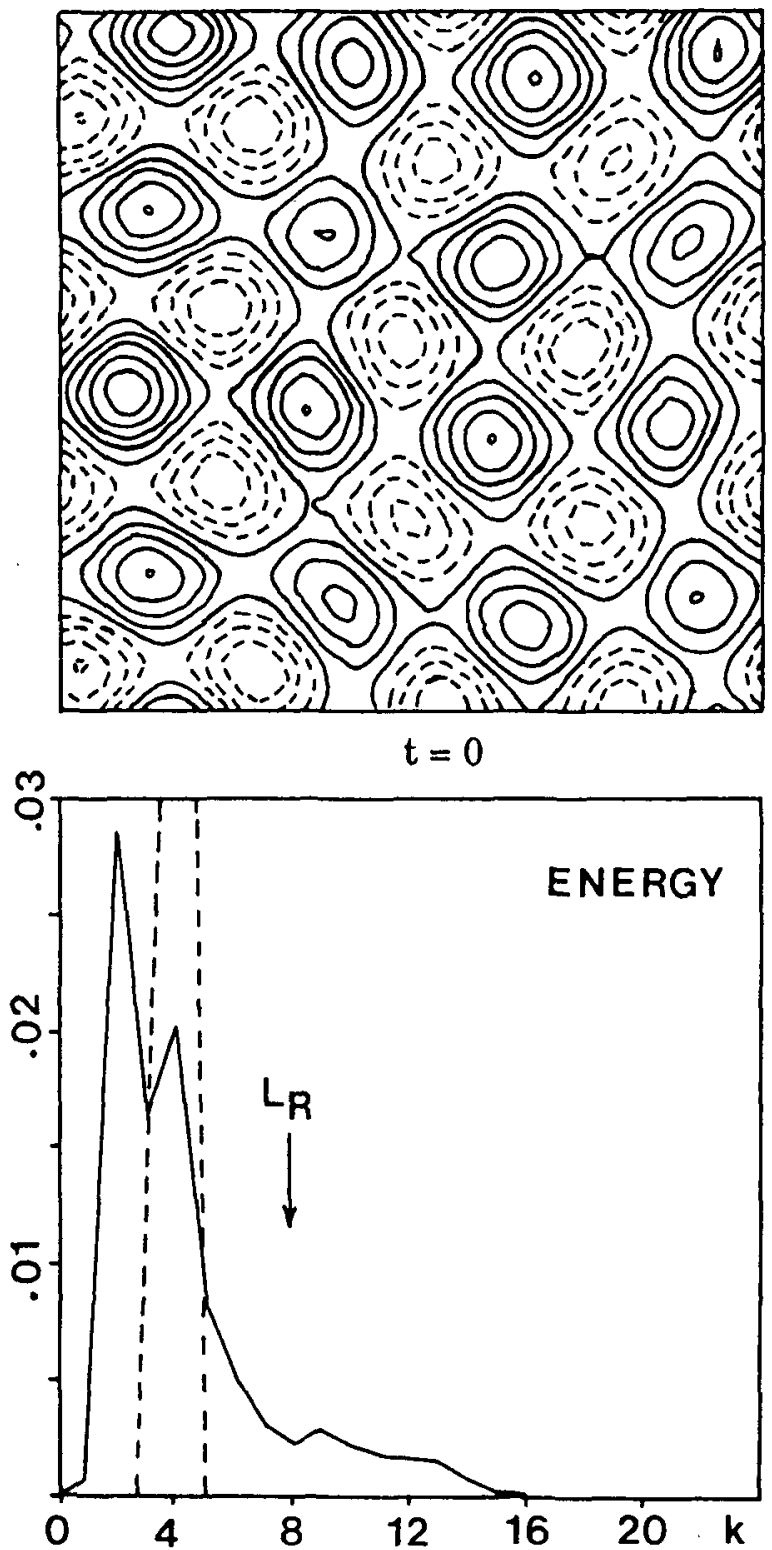

noted: emergence of eddies, selection of anticyclones, and bimodal character with frontal-geostrophic eddies evolving in a quasi-geostrophic background. The initial spectrum centered around $k=8\left(L=2.52 L_{R}\right)$ develops a peak from $k=4$ to $k=6\left(L=5 L_{R}\right.$ to $L=3.3 L_{R}$ or about the intermediate length scale $L=4 L_{R}$ $\left.=\alpha^{-1 / 3} L_{R}\right)$, and a tail extending from $k=12$ to $k$ $=30$ (centered on $k=20$ which corresponds to the deformation radius).

At the time when the computations of runs I and II were ended, a few eddies were still on the verge of or in the process of merging, but the general pattern of the $\eta$ field and the shape of its spectrum had not changed substantially over more than a decade of in-

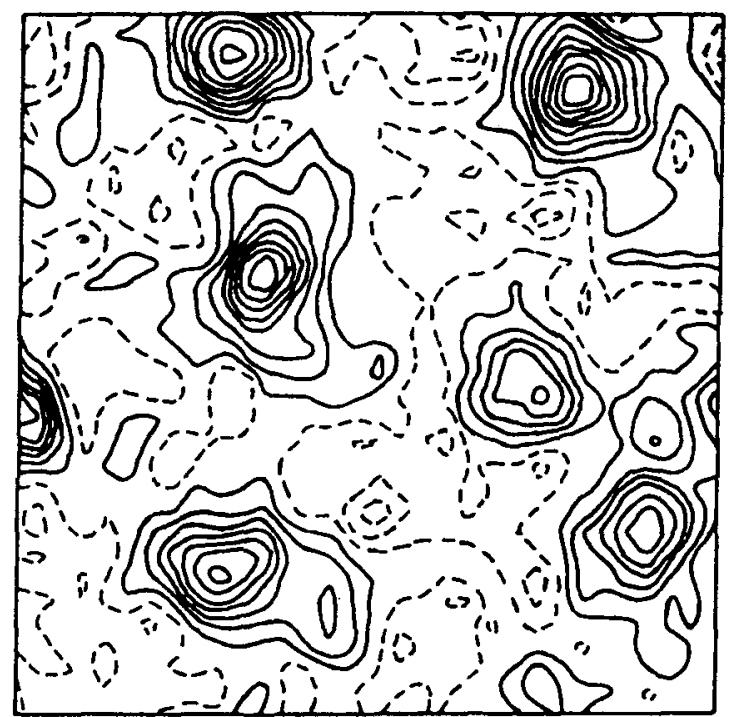

$\mathrm{t}=3750=94$ time scales
FIG. 5. Plots corresponding to run I of Fig. 4. The initial parameters are $L=2 L_{R}, \delta H=0.4 H$, and thus $E=0.10$ and the grid is $64 \times 64$. The upper panels compare the $\eta$ fields at the initial and final times ( $t=0$ and $t=3750$ or 94 intrinsic time scales). The lower panel displays the initial and final spectra. Note the emergence of a few large eddies, all being anticyclonic, the decrease in length scale in the turbulent background, and the peak-and-tail final spectrum. trinsic time scales. Hence, the plots displayed on Figs. 5 and 6 can be considered as those of a statistical equilibrium.

\section{A simple theory of bimodality}

The existence in the parameter space of a dividing line along which the energy cascade is arrested and a statistical equilibrium reached, calls for a theoretical explanation, and the observed dichotomy of that equilibrium suggests a simple model. Let us then assume a distribution of the interfacial displacement of $\eta$ consisting of regions (blobs) of anticyclonic eddies embedded in a turbulent background, the former covering the fraction $F$ of the total area, and the latter the 

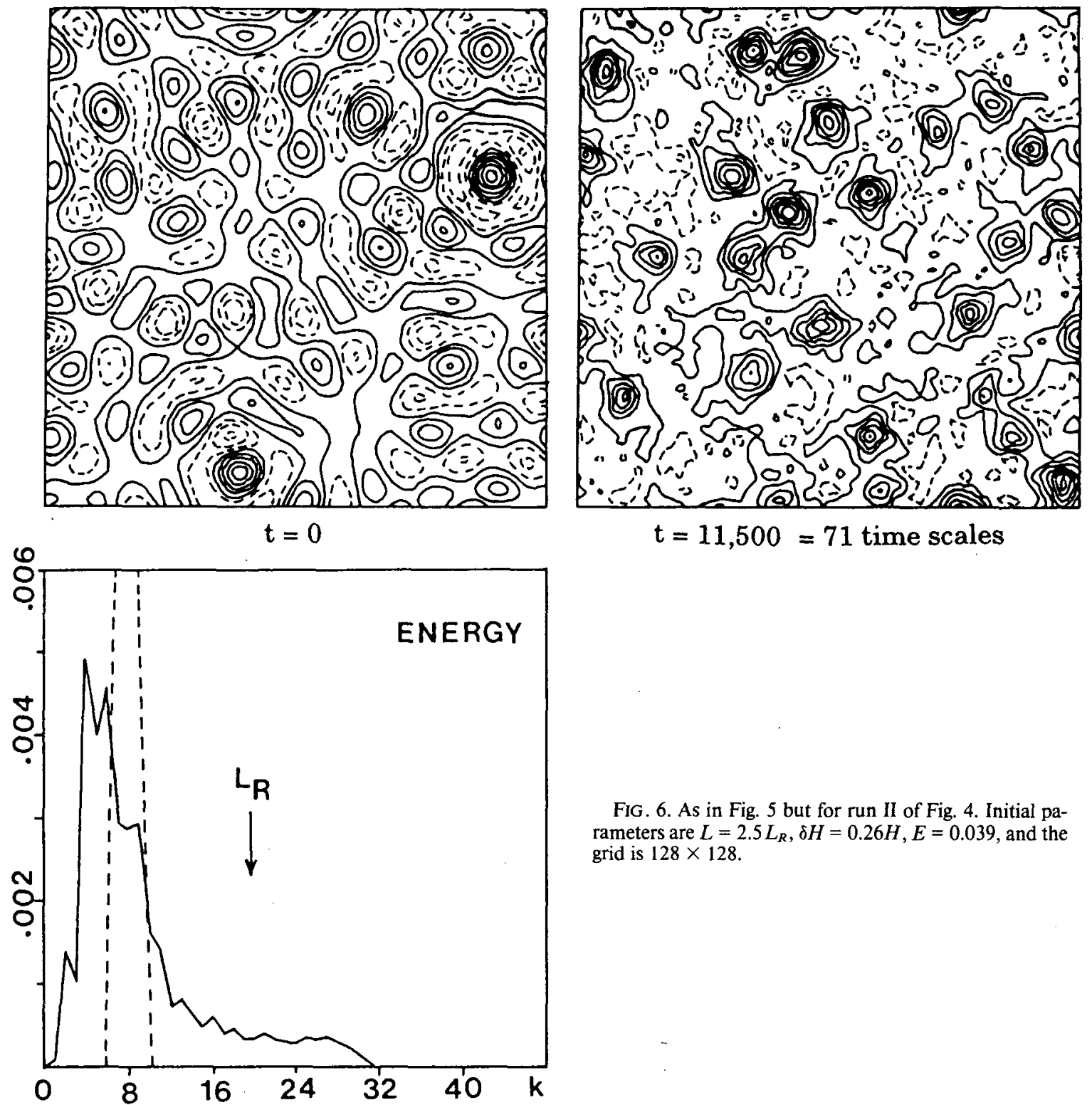

FIG. 6. As in Fig. 5 but for run II of Fig. 4. Initial parameters are $L=2.5 L_{R}, \delta H=0.26 H, E=0.039$, and the grid is $128 \times 128$.

complementary fraction $1-F$. In keeping with the numerical results, we further assign to the eddies a length scale $L_{1}=\alpha^{-1 / 3} L_{R}$ and an amplitude $\delta H_{1}=H$, while for the background we take $L_{2}=L_{R}$ and $\delta H_{2}$ $\ll H$, placing the eddies and the background turbulence in the frontal-geostrophic and quasi-geostrophic regimes, respectively. The fraction of area occupied by the eddies, $F$, and the amplitude of the background turbulence, $\delta \mathrm{H}_{2}$, are two undetermined quantities.

Since all eddies have the same sign, the conservation of mass requires

$$
F \delta H_{1}=(1-F) \delta H_{2},
$$

which simply states that the mean interfacial level is at $\eta=0$, and that the volume contained in the eddies

translates into an interfacial rise in the background. (Note that this statement does not take into account that the eddies are anticyclonic but only that they all have the same sign.) With the additional assumption that vertical interfacial excursions in the background are on the same order as the mean rise of the interface (in order not to multiply the number of unknowns and because the numerical results do not suggest otherwise), the total energy takes the form

$$
\begin{aligned}
E & =\frac{1}{2} F\left[\delta H_{1}^{2}+\frac{\delta H_{1}^{2}}{L_{1}^{2}}\right]+\frac{1}{2}(1-F)\left[\delta H_{2}^{2}+\frac{\delta H_{2}^{2}}{L_{2}^{2}}\right] \\
& =\frac{1}{2}\left(1+\alpha^{2 / 3}\right) F+(1-F) \delta H_{2}{ }^{2} .
\end{aligned}
$$


The first term is the eddy contribution while the second is that of the background. With the energy level set by the initial conditions, $E$ is considered as a known quantity, and Eqs. (28)-(29) form a two-by-two system of equations for $\mathrm{F}$ and $\delta \mathrm{H}_{2}$. Elimination of $\delta \mathrm{H}_{2}$ yields the following quadratic equation for $F$

$$
\left(1-\alpha^{2 / 3}\right) F^{2}+\left(1+\alpha^{2 / 3}+2 E\right) F-2 E=0,
$$

of which we must retain the positive root. (It is easily shown that this root is less than unity as long as $\alpha$ $<1$, which is the case.) Once $F$ is determined, $\delta H_{2}$ is obtained from

$$
\delta H_{2}=\frac{F}{1-F},
$$

and the average amplitude and length scale and can be derived in accordance with formulae (11) written as

$$
\begin{aligned}
& \delta H^{2}=F \delta H_{1}{ }^{2}+(1-F) \delta H_{2}{ }^{2}, \\
& \frac{\delta H^{2}}{L^{2}}=F \frac{\delta H_{1}{ }^{2}}{L_{1}{ }^{2}}+(1-F) \frac{\delta H_{2}{ }^{2}}{L_{2}{ }^{2}}
\end{aligned}
$$

to obtain

$$
\delta H=\left[\frac{F}{1-F}\right]^{1 / 2}, L=\left[F+\left(1-\alpha^{2 / 3}\right) F\right]^{-1 / 2}
$$

In summary, for a given ratio of deformation radius to planetary scale (quantity $\alpha$ ) and for a given energy level $(E)$, equation (30) provides the fraction of area occupied by the anticyclonic eddies, and formulae (31) and (33) provide the amplitude of disturbances in the turbulent background, the mean amplitude and the mean length scale. For example, run I corresponds to $\alpha=1 / 64$ and $E=0.01$, for which we find successively: $F=0.143,1-F=0.857, \delta H_{2}=0.167, \delta H=0.409$ and $L=2.26$. Hence, the prediction is that the anticyclonic eddies will ultimately occupy only $14 \%$ of the area, the amplitude of the background should be 0.167 , and the mean values be $L=2.26$ and $\delta H=0.409$. These values are quite on target, for an examination of Fig. 5 reveals that $15-20 \%$ of the area is occupied by eddies, the amplitude in the turbulent background is about 0.2 , and the initial values of $L$ and $\delta H$, which have remained almost unchanged throughout the calculations, were 2.0 and 0.40 , respectively. For run II $(\alpha=1 / 64$ and $E=0.039)$, the predictions are: $F$ $=6.5 \%, 1-F=93.5 \%, \delta H_{2}=0.069, \delta H=0.264$, and $L=2.85$, while the experimental values are $F=5$ to $10 \%, \delta H_{2}=0.06$ to $0.10, \delta H=0.250$ and $L=2.52$. We note that a reduction in the energy level leads to a reduction in the fraction of area occupied by eddies, and that the numerical results (compare Figs. 5 and 6 ) do indeed reveal such a tendency. Finally and for completeness we evaluate the contributions of the an- ticyclonic eddies and turbulent background to the total energy; for run I, the eddies contribute $76 \%$ and the background $24 \%$, while for run II the eddies contribute $88 \%$ and the background $12 \%$ of the energy.

Fixing the value of $\alpha(\alpha=1 / 64$ for the present numerical experiments), we can vary the energy level and track the point $(L, \delta H)$ in the parameter space. By so doing, we obtain the $S$-shape curve of Fig. 4, along which the statistical equilibrium should be found, and we note a high degree of agreement between this curve and the set of points obtained from the numerical experiments. In other words, the above simple theory seems to capture the essence of the statistical equilibrium or, put another way, the characteristics of the statistical equilibrium can be explained solely with the principles of conservation of mass and energy and with the knowledge of the existence of a bimodal distribution.

\section{On the selection of anticyclonic eddies}

The main question at this stage concerns the process (or processes) behind the selection of anticyclonic eddies. Simply put: why do anticyclones merge and cyclones break up? Although a definite answer has yet to be formulated, a few elements can be advanced to suggest that anticyclonic eddies are generally more robust and merge more freely than their cyclonic counterparts.

The same spectral model as described above was used for another series of single-eddy experiments. Initial conditions consisted of an isolated circular vortex, and the parameters which varied from run to run were its radius, amplitude and polarity. Each run showed quite unambiguously that the eddy either kept its identity for a large number of intrinsic time scales despite changes such as a westward drift and a wake formation, or broke up within a few intrinsic time scales. Figure 7 contrasts two examples, while Fig. 8 recapitulates the results for all runs.

From these results, several conclusions can be drawn. First, the disparity between cyclones and anticyclones is most noticeable when the symmetry-breaking terms are important $(\delta H \sim H)$, i.e., when the frontal effects enter the dynamics. This comes as no surprise; however, another region of disparity also exists for weaker eddies. In the parameter space, this region forms a band loosely aligned with the Rhines scale. Finally and perhaps most importantly, the results demonstrate that cyclonic eddies are capable of self destruction. In other words, interactions with the surrounding eddies and turbulence are not necessary to account for the absence of cyclonic eddies in the statistical equilibrium. The single-eddy experiments of Matsuura and Yamagata (1982) were the first to indicate that anticyclones are relatively more robust than cyclones when the frontal correction is retained in the beta effect (quadratic nonlinearity). Without providing a thorough explanation, 


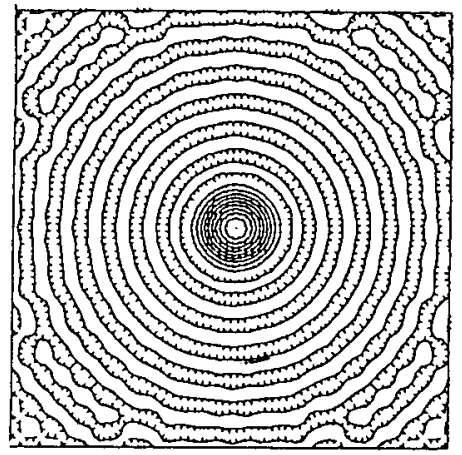

$t=0$

(a)

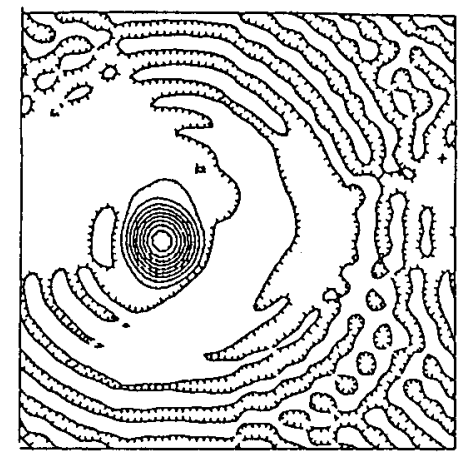

(b)

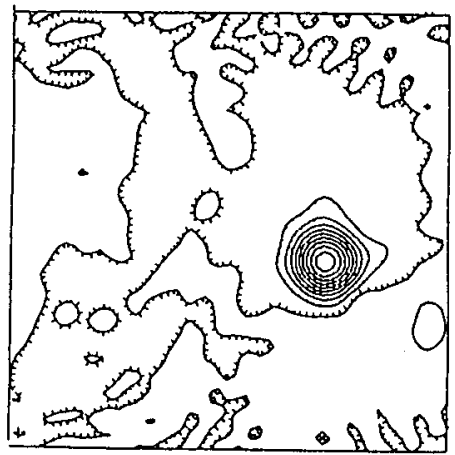

$\mathrm{t}=1000 \quad$ (c)

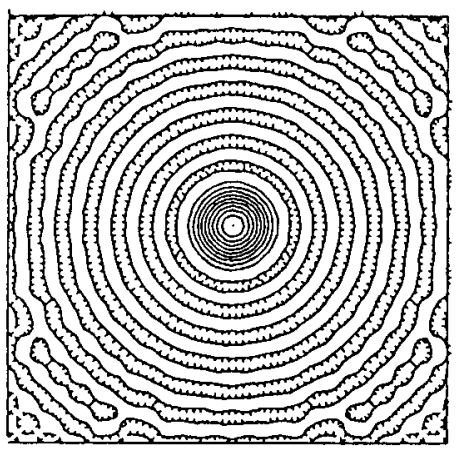

$t=0$ (d)

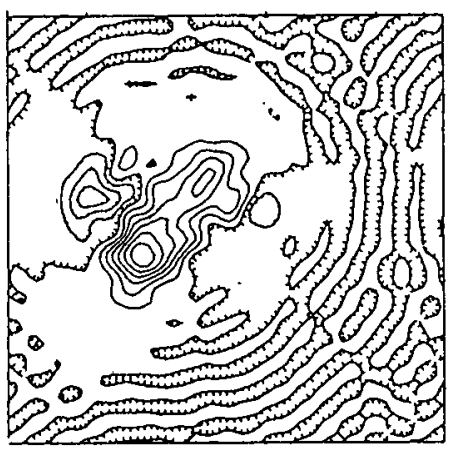

(e)

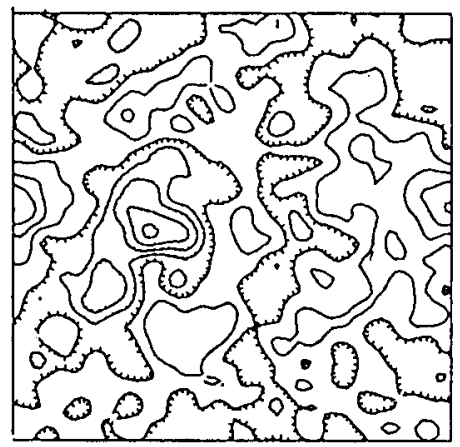

$t=1000$

(f)

FIG. 7. Evolution of an anticyclone (upper row) and cyclone (lower row). Except for the sign difference, each initial eddy has amplitude $\delta H=1.0 H$ and radius $R=3.0 L_{R}$. Note the survival of the anticyclone, despite a substantial westward drift, and the self-destruction of the cyclone.

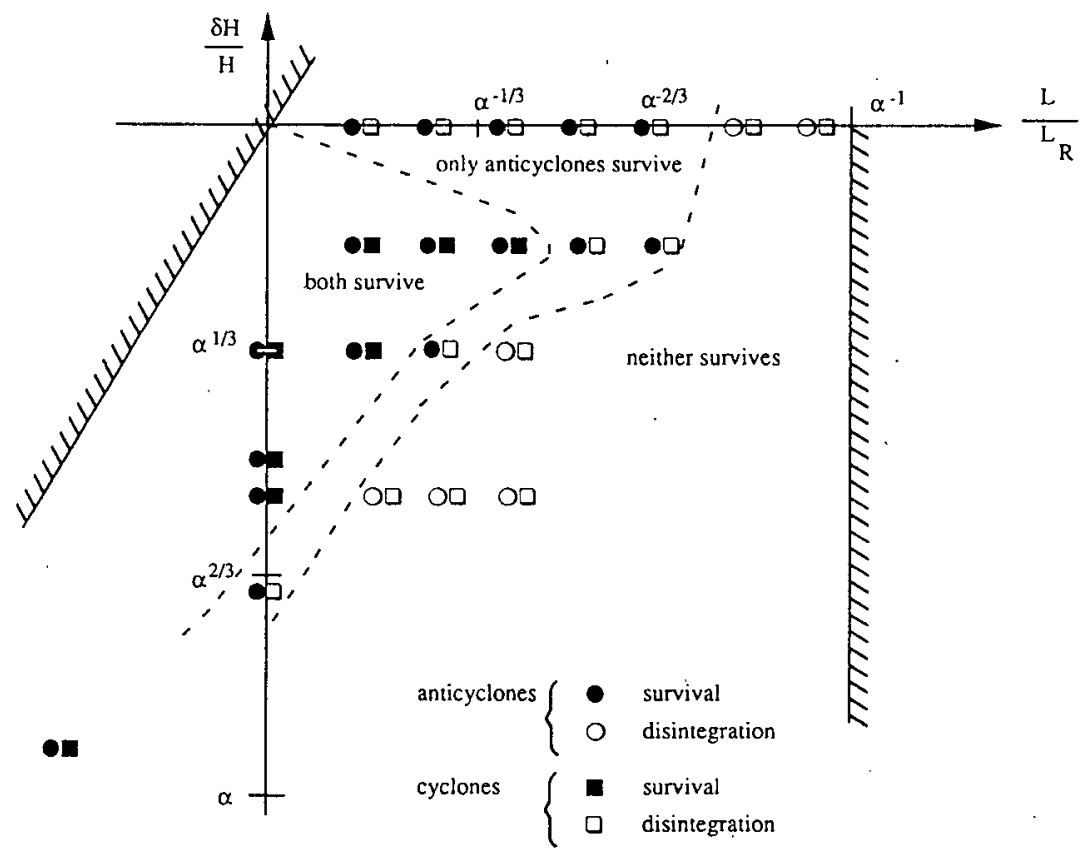

FIG. 8. Summary chart of numerical experiments involving single initial eddies. Note the disparity between anticyclones and cyclones when frontal effects are important $(\delta H / H \sim 1)$. 
Sutyrin and Yushina (1986), too, noted that, in their nonquasi-geostrophic numerical experiments, cyclonic eddies tend to interact much more slowly and merge much less frequently than anticyclonic eddies of similar size and amplitude.

A first candidate theory to explain the asymmetry between long-lived anticyclones and self-dispersing cyclones relies on the quadratic correction in the beta term. Matsuura and Yamagata (1982) showed that, at relatively long scales, an isolated vortex is, at first-order, subjected to a simple westward translation and, at the next order, governed by a $K d V$-type equation. In this latter equation, the nonlinear steepening stems from the quadratic beta term and balances dispersion only if the vortex is anticyclonic. Cyclones perish by combined nonlinear steepening and dispersion. In other words, anticylones are long-lived and cyclones are not, at those long length scales.

This argument, based on the quadratic correction in the beta term, cannot however account for the asymmetry between cyclones and anticyclones in the vicinity of the deformation radius $\left(\delta H \sim H, L>L_{R}\right)$. At those scales, the beta effect is secondary, and the asymmetry should find its origin in the cubic nonlinearities of the Jacobian term, which are the only other sources of symmetry breaking in the governing equation. Figure 9 shows that an $f$-plane, frontal cyclonic vortex disintegrates into a number of smaller vortices; with identical radius and amplitude, the corresponding anticyclone remains unchanged. Following is a tentative stability argument suggesting that, on an $f$-plane, frontal dynamics yield stable anticyclones and unstable cyclones.

With $\beta_{0}=0$ (hence $\alpha=0$ ), the governing equation (5) in terms of the total layer depth $h=1+\eta$ becomes

$$
\frac{\partial h}{\partial t}=J\left(h, h \nabla^{2} h+\frac{1}{2}|\nabla h|^{2}\right)
$$

and has the following invariants

Moments of volume: $I_{n}=\iint h^{n} d x d y$

$$
(n=1,2, \cdots)
$$

Angular momentum: $A=\frac{1}{2} \iint r^{2} h d x d y$

$$
\left(r^{2}=x^{2}+y^{2}\right)
$$

Kinetic energy: $\quad K=\frac{1}{2} \iint h|\nabla h|^{2} d x d y$.

[In frontal dynamics (Cushman-Roisin 1986), the primary contribution to the energy is the potential energy, proportional to $I_{2}$, which is conserved on both the $f$-and $\beta$-planes. That the kinetic energy is a separate invariant is a particularity of the $f$-plane case.] Let us now investigate the states that optimize the value of $K$ for given values of the invariants $I_{n}$ and $A$. Forming the functional

$$
\begin{aligned}
F(h)=\iint\left[\frac{1}{2} h|\nabla h|^{2}+\sum_{1}^{\infty} \lambda_{n} h^{n}+\frac{1}{2} \omega r^{2} h\right] \\
\times d x d y-\sum_{1}^{\infty} \lambda_{n} I_{n}-\omega A,
\end{aligned}
$$

one finds that its first variation vanishes if $h$ obeys the equation

$$
h \nabla^{2} h+\frac{1}{2}|\nabla h|^{2}=\sum_{1}^{\infty} n \lambda_{n} h^{n-1}+\frac{1}{2} \omega r^{2},
$$

or, after the application of the operator $J(h, \quad)$,

$$
J\left(h, h \nabla^{2} h+\frac{1}{2}|\nabla h|^{2}\right)=J\left(h, \frac{1}{2} \omega r^{2}\right) .
$$

A comparison between Eqs. (34) and (35) reveals that the solutions to the latter are the steady solutions to the former $(\omega=0, \partial h / \partial t=0)$ or those solutions that

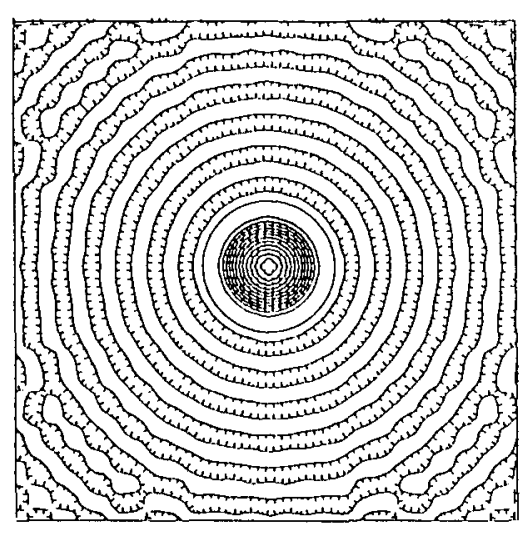

$t=0$

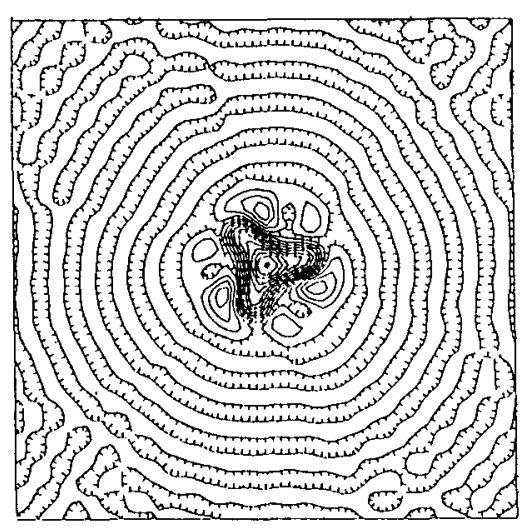

$t=522$

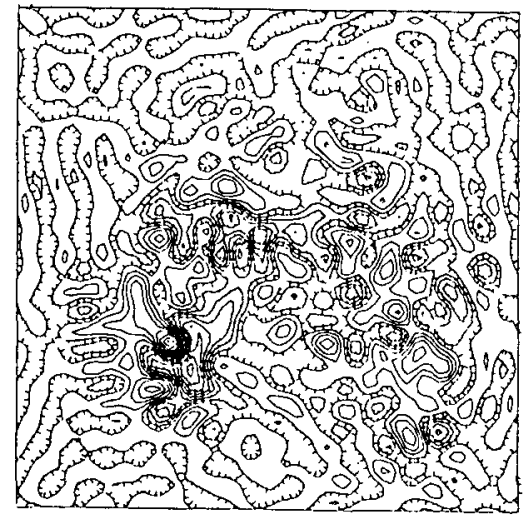

$t=1002$

FIG. 9. Evolution of a frontal cyclonic vortex $\left(\delta H=H, L=3 L_{R}\right)$ on an $f$-plane. Note the disintegration of the feature. The anticyclonic vortex of same amplitude and size (not shown) remained unchanged over the same time interval. 
steadily rotate without change in shape $(\omega \neq 0, \omega=$ angular frequency of precession). Therefore, steady solutions, such as all axisymmetric eddies, are amongst those that optimize the value of $K$. The second variation of $K$ is

$$
\delta^{2} K=\iint\left[\frac{1}{2} h|\nabla \delta h|^{2}-\nabla^{2} h(\delta h)^{2}\right] d x d y .
$$

For anticyclonic vortices, $\nabla^{2} h$ is negative and $\delta^{2} K$ is positive definite, implying that the value of $K$ is a minimum for any steady anticyclone. In other words, steady anticyclonic eddies are stable. For cyclonic vortices, $\nabla^{2} h$ is positive and $\delta^{2} K$ has no definite sign implying that steady cyclones may correspond to saddle points of $K$ and therefore be unstable.

In the limit of weak, quasi-geostrophic vortices $(h$ $=1+\eta, \eta \ll 1)$, the first term in $\delta^{2} K$ dominates and hence $\delta^{2} K$ is positive definite. This implies, as it is well known, that circular quasi-geostrophic anticyclones as well as cyclones are stable, but it also implies that finite amplitudes (frontal effects, $\eta=0(1)$ ) are necessary to render cyclones unstable.

\section{Summary and discussion}

From the desire to explore the properties of geostrophic turbulence beyond the radius of deformation, the need arises to construct an equation enlarging somewhat the classical quasi-geostrophic formalism. Such a generalized geostrophic equation has been derived here. Although it has previously appeared in several other, similar but not identical, forms, the bounds of validity, the physical contents and the invariants of this equation have been examined here in some detail. The single most important addition to the physical contents of the formalism is the retention of frontal effects at scales longer than the deformation radius. Namely, at such scales vertical displacements are not restricted in amplitude. Overall, the generalized equation is a hybrid equation comprising both the classical quasi-geostrophic and the so-called frontal-geostrophic formalisms.

The equation conserves volume and energy. It does not exactly conserve any enstrophy-like quantity, although every one of its reduced forms does. One remedy to the lack of conservation of enstrophy would be to resort to the use of the primitive governing equations, which is an impractical alternative in the study of geostrophic turbulence, at least at the present time, or to use a system of coupled prognostic-diagnostic equations (Salmon 1985; Sutyrin and Yushina 1986). Despite new terms, including a cubic nonlinearity, the equation is amenable to the numerical methods developed for the classical quasi-geostrophic equation.

For length scales shorter than and up to the deformation radius, the classical properties of geostrophic turbulence are recovered, namely the cascade of energy toward larger scales, the cascade of enstrophy toward shorter scales, and the emergence of a few large eddies.
Physically, the scenario is as follows: for some reason, perhaps still beyond our understanding, merging of like-sign vortices is a favored activity, and each merging entails a certain amount of filamentation and concludes with the lumping of energy in a new, larger vortex (McWilliams 1984). The enstrophy cascade is then interpreted as nothing else than repeated filamentation induced by merging events, while the energy cascade is the expression of the increased coherency resulting from the formation of ever larger eddies.

For scales longer than the deformation radius, frontal effects add new physical constraints and possibilities, and two new, major phenomena are noted. First, the energy cascade toward longer scales, reaches a saturation, and geostrophic turbulence halts at a statistical equilibrium. Second, the emerging eddies are all anticyclonic. This very systematic discrimination among vortices can only be explained by the frontal effects since these, and only these, break the symmetry between cyclones and anticyclones. But why this lack of symmetry provokes such a unilateral selection and why anticyclones are favored over cyclones are two questions that still need to be answered. Some suggestions were offered in section 8 .

A simple theory of bimodality, whereby the turbulent field is assimilated to a mixture of frontal-geostrophic anticyclonic vortices embedded in a quasi-geostrophic turbulent background, predicts the characteristics of the statistical equilibrium such as average length scale, average amplitude, fraction of area occupied by anticyclonic vortices, and energy partition between the vortices and the turbulent background. These theoretical predictions closely agree with the numerical findings.

We note that the length scale at which the statistical equilibrium is reached has no direct relation to the Rhines scale (where a balance is struck between nonlinear advection and Rossby-wave propagation ). The length scale rather betrays a saturation in the rate of eddy merging (if the turbulence originated from shorter scales) or of eddy breaking (if the turbulence originated from longer scales) at a time when the available mass and energy is divided among anticyclonic frontal vortices and a turbulent field at the scale of the deformation radius.

Acknowledgments. The authors are deeply indebted to Drs. J. C. McWilliams, V. D. Larichev, G. P. Williams, and T. Yamagata for most pertinent comments and helpful suggestions. Support was provided under Contract N00014-87-J-1209 with the Office of Naval Research and from the Supercomputer Computations Research Institute at Florida State University where calculations were performed on a Cyber-205.

\section{REFERENCES}

Basdevant, C., B. Legras, R. Sadourny and M. Beland, 1981: A study of barotropic model flows: Intermittency, waves and predictability. J. Atmos. Sci., 38, 2305-2326.

Charney, J. G., and G. R. Flierl, 1981: Oceanic analogues of large 
scale atmospheric motions. Evolution of Physical Oceanography, B. A. Warren and C. Wunsch, Eds., The MIT Press, Chap. 18, 504-548.

Cushman-Roisin, B., 1986: Frontal geostrophic dynamics. J. Phys. Oceanogr., 16, 132-143.

bulence beyond the radius of deformation. Mesoscale/Synoptic Coherent Structures in Geophysical Turbulence, J. C. J. Nihoul and B. M. Jamart, Eds., Elsevier Oceanogr. Ser. 50, 51-74.

Fornberg, B., 1977: A numerical study of 2-D turbulence. J. Comput. Phys., 25, 1-31.

Gent, P. R., and J. C. McWilliams, 1983a: Consistent balanced models in bounded and periodic domains. Dyn. Atmos. Oceans, 7, 6793.

$\longrightarrow$, and — $1983 \mathrm{~b}$ : Regimes of validity for balanced models. Dyn. Atmos. Oceans, 7, 167-183.

Hukuda, H., and T. Yamagata, 1988: A unified geostrophic equation with application to a cold core ring. Tellus, 40A, 407-418.

Larichev, V. D., 1985: On free evolution of barotropic geostrophic turbulence. Oceanology, 85, 425-430. (English translation).

- 1989: Differential rotation (beta effect) as an organizing factor in mesoscale dynamics. Mesoscale/Synoptic Coherent Structures in Geophysical Turbulence, J. C. J. Nihoul and B. M. Jamart, Eds., Elsevier Oceanography Series, 50, 41-49.

Matsuura, T., and T. Yamagata, 1982: On the evolution of nonlinear planetary eddies larger than the radius of deformation. J. Phys. Oceanogr., 12, 440-456.

Meiss, J. D., and W. Horton, 1983: Solitary drift waves in the presence of magnetic shear. Phys. Fluids, 26, 990-997.

McWilliams, J. C., 1983: On the relevance of two-dimensional tur- bulence to geophysical fluid motions. J. Mec. Theor. Appl. (Special issue), 83-97.

- 1984: The emergence of isolated coherent vortices in turbulent flow. J. Fluid Mech., 146, 21-43.

Mirabel, A. P., and A. S. Monin, 1980: Geostrophic turbulence (A review). Izv. Atmos. Ocean. Phys., 16, 749-757 (English translation).

Petviashvili, V. I., 1980: Red Spot of Jupiter and drift solitons in a plasma. JETP Lett., 32, 619-622.

Rhines, P. B., 1975: Waves and turbulence on a beta-plane. J. Fluid Mech., 69,417-443.

-_, 1979: Geostrophic turbulence. Ann. Rev. Fluid Mech., 11, 401441.

Salmon, R., 1985: New equations for nearly geostrophic flow. $J$. Fluid Mech., 153, 461-477.

Sutyrin, G. G., and I. G. Yushina, 1986: Interaction of synoptic eddies of finite amplitude. Dokl. Akad. Nauk SSSR, 288(3), 585-589 (Russian text) or 20-24 (English translation).

Weinstein, A., 1983: Hamiltonian structure for drift waves and geostrophic flow. Phys. Fluids, 26, 388-390.

Williams, G. P., 1985: Geostrophic regimes on a sphere and a beta plane. J. Atmos. Sci., 42, 1237-1243.

- , and T. Yamagata, 1984: Geostrophic regimes, intermediate solitary vortices, and Jovian eddies. J. Atmos. Sci., 41, 453478.

, and R. J. Wilson, 1988: The stability and genesis of Rossby vortices. J. Atmos. Sci., 45, 207-241.

Yamagata, T., and S. Umatani, 1990: The response of the eastern tropical Pacific to seasonal winds: The Costa Rica Dome. Proc. 21st Int. Liège Colloq., Elsevier Oceanogr. Ser. 